05

\title{
Эффективная генерация характеристического рентгеновского излучения при воздействии чирпированных фемтосекундных лазерных импульсов на медную мишень при локальном поддуве гелия
}

\author{
(C) А.А. Гарматина ${ }^{1}$, М.М. Назаров ${ }^{1}$, П.А. Щеглов ${ }^{1}$, М.В. Чащин ${ }^{1}$, В.А. Алешкевич ${ }^{2}$, Б.Г. Бравый ${ }^{3}$, \\ В.М. Гордиенко ${ }^{2}$, В.Я. Панченко ${ }^{1}$ \\ ${ }^{1}$ НИЦ „Курчатовский институт“, \\ 123182 Москва, Россия \\ ${ }^{2}$ Физический фракультет МГУ им. М.В. Ломоносова, \\ 119992 Москва, Россия \\ ${ }^{3}$ Департамент нанофотоники, Институт проблем химической физики, \\ 142432 Черноголовка, Россия \\ e-mail: alga009@mail.ru
}

Поступила в редакцию 01.11.2021 г.

В окончательной редакции 12.12.2021 г.

Принята к публикации 30.12.2021 г.

Разработан и создан эффективный лазерно-плазменный источник спектрально яркого рентгеновского излучения. Сочетание режима управления длительностью интенсивных фемтосекундных лазерных импульсов и гелиевого поддува в область микроплазмы, зажигаемой на медной мишени, находящейся в атмосферных условиях, позволяет оптимизировать условия эффективной генерации рентгеновского излучения сверхкороткой длительности и достижения максимального потока $\mathrm{K}_{\alpha}$-фотонов $2 \cdot 10^{7} \mathrm{ph} / \mathrm{pulse} / 2 \pi \mathrm{sr}$ с эффективностью конверсии $10^{-5}$.

Ключевые слова: фемтосекундные лазерные импульсы, рентгеновское излучение.

DOI: $10.21883 /$ OS.2022.04.52266.64-21

\section{Введение}

Рентгеновское излучение в настоящее время находит все более широкое применение в физике твердого тела, кристаллографии, микроскопии, спектроскопии, визуализации и в других аналитических задачах [1-3]. Существенно, что в работах, ориентированных на исследования конденсированных сред, находящихся в неравновесных состояниях, часто рассматривают лишь относительно низкочастотные атомные смещения. Сверхбыстрая дифракция относится к семейству методов, известных как накачка-зондирование, в которых реализованы два синхронизированных импульса. При этом в качестве накачки может быть использован фемтосекундный лазерный импульс, ответственный за инициирование структурных изменений в процессе создания неравновесного состояния. Пробное излучение в этом случае представляет собой ультракороткий рентгеновский импульс, который рассеивается от облученного образца для создания дифракционной картины. Достижения в сверхбыстрой структурной динамике зависят от эффективности генерации сверхкоротких импульсов жесткого спектрально яркого рентгеновского излучения. Возникает также необходимость осуществления прецизионной синхронизации между импульсами зондирования и накачки. Отметим, что для реализации атомного пространственного разрешения, как правило, требуется относительно жесткое рентгеновское излучение (с энергией рентгеновских фотонов более $6 \mathrm{keV}$ ), свободно распространяющееся в воздухе. Для решения задач, ориентированных на нестационарный структурный анализ и на получение дифракционной картины за малое (единицы пикосекунд) время, необходим лазерный микроплазменный источник с высоким выходом характеристических рентгеновских фотонов - около $10^{8} \mathrm{ph} / \mathrm{s}[1,3]$. Обычно, лазерно-плазменные источники рентгеновского излучения получаются при фокусировке излучения мощных импульсных лазеров на мишень, расположенную в вакуумной камере [3]. Этот подход имеет ряд недостатков, которые связаны с неизбежным загрязнением камеры разлетающимися продуктами абляции мишени, громоздкими размерами камеры и сложной автоматизацией. Поэтому гораздо удобнее использовать источник, расположенный вне вакуумной камеры. При этом наличие газовой среды перед мишенью будет предотвращать разлет продуктов абляции и загрязнение фокусирующей оптики и иных устройств.

В воздухе при достижении порога пробоя лазерным излучением среда ионизуется с образованием плазмы, изменяется показатель преломления среды и возникают условия для дефокусировки, рассеяния и потерь энергии лазерного пучка. Все эти факторы приводят к ограничению доставляемой на мишень интенсивности, а значит, 
понижают выход рентгеновского излучения. Порог плазмообразования среды можно увеличить за счет замены воздуха на газ с большим потенциалом ионизации, например, на гелий [4]. Это позволяет увеличить доставляемую интенсивность и повысить выход рентгеновского излучения при создании лазерно-индуцированного микроплазменного источника. Так, в [4] локальный поддув гелия в область перетяжки в сочетании с острой фокусировкой фемтосекундного лазерного пучка с апертурой NA 0.4 позволил достичь выхода рентгеновских $\mathrm{K}_{\alpha}$-фотонов меди $(8 \mathrm{keV})$ около $2 \cdot 10^{7} \mathrm{ph} / \mathrm{pulse} / 2 \pi \mathrm{sr}$. Но такую схему сложно использовать для практических применений, поскольку при малой длине перетяжки рентгеновский сигнал слишком чувствителен к неидеальному расположению мишени в фокальной плоскости. Также возникают пространственные ограничения на размещение опто-механических элементов в используемой схеме. Например, рентгеновской оптики.

Схема с более мягкой фокусировкой и, следовательно, большей длиной перетяжки позволит создать лазерноплазменный источник рентгеновского излучения, менее чувствительный к положению мишени в фокальной плоскости. В работе [5] нами было теоретически показано и экспериментально подтверждено, что использование чирпированных импульсов в схеме с NA $\sim 0.1$ обеспечивает увеличение выхода рентгеновского излучения. Отметим, что тенденция на увеличение выхода рентгеновского излучения при использовании чирпированных фемтосекундных лазерных импульсов ранее нами была также обнаружена в экспериментах с использованием фемтосекундного филамента, воздействующего на металлическую мишень, расположенную в воздухе [6].

Предметом настоящей работы является разработка нового подхода к эффективной генерации спектральнояркого рентгеновского излучения сверхкороткой длительности на базе твердотельного лазерно-плазменного источника. Использование титан-сапфировой лазерной системы, генерирующей широкополосные импульсы с соответственным варьированием длительности в фемтопикосекундном диапазоне, позволит понизить ионизационные потери, „обойти“ эффект ограничения интенсивности и достичь такого уровня плотности энергии, при котором поток характеристических рентгеновских фотонов достигнет $10^{8} \mathrm{ph} / \mathrm{s}$. Это отвечает минимальным требованиям для большинства приложений.

\section{Критерии острой фокусировки}

Эффективный метод генерации рентгеновского излучения при нормальных атмосферных условиях предполагает минимизацию негативного влияния лазерно индуцированной преплазмы в приповерхностной зоне мишени на интенсивность фемтосекундного лазерного излучения на самой мишени. Ослабление интенсивности, во-первых, происходит из-за потерь на ионизацию плазмы и, во-вторых, из-за нелинейной дефокусировки

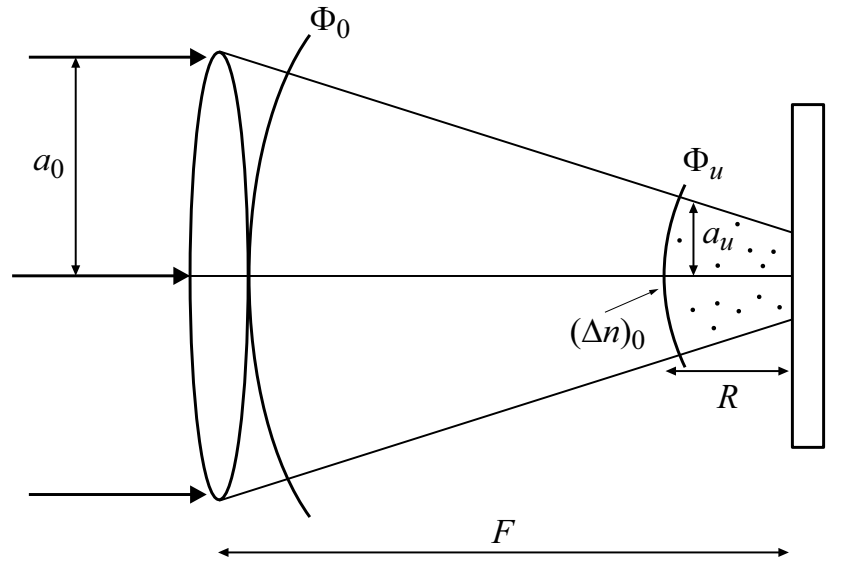

Рис. 1. Схема взаимодействия сфокусированного лазерного пучка с мишенью в условиях ионизации.

лазерного пучка в плазме. Для достижения оптимальных условий лазерного воздействия необходимо использование фокусирующей оптики с высокой числовой апертурой. При этом старт процесса ионизации может быть замедлен, а длина ионизированного пути может быть соответственно уменьшена за счет увеличения порога ионизации рабочей среды, например, при использовании гелия [4]. В настоящей работе с помощью аналитических оценок сформулирован критерий выполнения режима острой фокусировки, при котором может подавляться плазменная дефокусировка пучка.

Падающий на линзу с фокусным расстоянием $F$ коллимированный гауссов световой пучок с амплитудой $A(r)=A_{0} \exp \left(-r^{2} / a_{0}^{2}\right)$ приобретает искривленный после линзы фазовый фронт $\Phi_{0}(r)=k r^{2} /(2 F)$ (рис. 1).

Будем считать, что эффективное образование плазмы происходит при некоторой интенсивности на оси пучка $I_{u 0}$. Это произойдет при достижении пучком радиуса $a_{u}$, который определяется из закона сохранения энергии $I_{0} a_{0}^{2}=I_{u 0} a_{u}^{2}$, где $I_{0}-$ интенсивность на оси падающего на линзу пучка, $R$ - радиус кривизны волнового фронта пучка, радиус $a_{u}$ определяется из соотношения $\frac{a_{0}}{F}=\frac{a_{u}}{R}$. Соответственно фазовый фронт пучка на входе в плазменную среду описывается в этом сечении выражением

$$
\Phi_{u}=\frac{k r^{2}}{2 R} .
$$

При ионизации приращение показателя преломления плазмы $\Delta n$ к окончанию действия импульса в этом же сечении можно записать в виде

$$
|\Delta n|=\mathrm{const} \cdot I^{\gamma}=(\Delta n)_{0} \exp \left(-\frac{2 \gamma r^{2}}{a_{u}}\right),
$$

где $\gamma-$ кратность многофотонной ионизации. При приближении к фокусу линзы фазовый набег из-за самодефокусировки составит

$$
\Phi_{n l}=k \int_{0}^{R}|\Delta n| d z .
$$


Для оценки вынесем $\Delta n$ из под знака интеграла, поскольку протяженность плазменной среды невелика. Тогда

$$
\Phi_{n l}=k|\Delta n| R \approx k(\Delta n)_{0} R \exp \left(-\frac{2 \gamma r^{2}}{a_{u}^{2}}\right) .
$$

Очевидно, что при $\Phi_{u}>\Phi_{n l}$ дефокусировка не влияет заметно на распространение пучка. Для сравнения (1) и (2) запишем последнее выражение для приосевой области:

$$
\Phi_{n l}=k(\Delta n)_{0} R\left(1-\frac{2 \gamma r^{2}}{a_{u}^{2}}\right) .
$$

Постоянный фазовый набег не оказывает влияния на дефокусировку пучка, поэтому условие слабого влияния нелинейной дефокусировки примет вид

$$
k r^{2} /(2 R)>k(\Delta n)_{0} R\left(2 \gamma r^{2} / a_{u}^{2}\right)
$$

Воспользовавшись параметром $\mathrm{NA}=\left(a_{u} / R\right)=(a / F)$, окончательно получим

$$
\mathrm{NA}>2\left(\gamma(\Delta n)_{0}\right)^{1 / 2}
$$

Последнее выражение может рассматриваться как критерий для определения оптимального режима острой фокусировки, при котором нелинейная дефокусировка в плазме мала. Полная однократная ионизация (соответствующая концентрации электронов $n=2.5 \cdot 10^{19} \mathrm{~cm}^{-3}$ ) приводит на длине волны $0.8 \mu \mathrm{m}$ к изменению показателя преломления на величину $\Delta n_{0}=7 \cdot 10^{-3}$. В предположении, что оптимальной средой, окружающей приповерхностный слой мишени, является гелий, имеющий потенциал ионизации $24.5 \mathrm{eV}$ (для гелия на данной длине волны $\gamma=16$ ), оптимальная острая фокусировка реализуется при условии NA $>0.6$.

В экспериментах такая числовая апертура достигается при использовании специальных короткофокусных линзовых объективов, через которые сложно транспортировать энергию более 2-3 mJ без искажения спектра лазерного излучения. Либо необходимо использовать лазерные пучки большой апертуры, которые нуждаются в корректирующей адаптивной оптике и требуют высокое качество изготовления фокусирующего устройства. Отметим, что экспериментальные исследования, выполненные в [4] с использованием фокусирующей оптики с числовой апертурой $\mathrm{NA}=0.4$, не позволяли выйти на предполагаемый оптимальный режим генерации рентгеновского излучения. Кроме того, релеевская длина при больших значениях NA становится столь малой, что возможные дефекты поверхности мишени уже заметно влияют на стабильность рентгеновского сигнала при генерации микроплазмы. Это может стать существенным для приложений, связанных, например, с задачами рентгеновской дифракции.

Ниже обсуждаются результаты экспериментов по реализации вневакуумного подхода для эффективной лазерно индуцированной генерации рентгеновского излучения в режиме „мягкой“ фокусировки. Он опирается на сочетании процесса поддува гелия, обладающего высоким потенциалом ионизации и позволяющего минимизировать число индуцированных электронов, и режима чирпированных импульсов для оптимизации доставки энергии в область взаимодействия лазерного излучения с веществом.

\section{Экспериментальная установка}

В экспериментах использовался фемтосекундный лазерный комплекс Ti:Sa c двумя каскадами усиления, длительность спектрально ограниченного импульса $30 \mathrm{fs}$ на длине волны $800 \mathrm{~nm}$, энергия в импульсе не превышала $6 \mathrm{~mJ}$ в данных экспериментах, частота следования импульсов $10 \mathrm{~Hz}$. Длительность импульса изменялась за счёт изменения взаимного расположения решёток в оптическом компрессоре и контролировалась методом SHG-FROG. Лазерный пучок диаметром $1 \mathrm{~cm}$ по уровню FWHM фокусировался внеосевой параболой $(\mathrm{NA}=0.1)$, что обеспечивало „мягкий“ режим фокусировки на движущуюся поверхность медной мишени. Перемещение мишени производилось с помощью вертикально и горизонтально ориентированных шаговых двигателей. Скорость перемещения мишени подбиралась таким образом, чтобы каждый последующий импульс взаимодействовал со „свежей“ п поверхностью мишени, размещенной в воздухе. Для подачи гелия в зону взаимодействия лазерного излучения с мишенью была изготовлена небольшая камера в виде отрезка силиконовой трубки, ориентированного открытыми частями к мишени и соответственно к падающему лазерному пучку. Заполнение камеры гелием проводилось с помощью иглы от шприца диаметром $0.4 \mathrm{~mm}$, острие которой располагалось в зоне возникновения плазмообразования в приповерхностной области мишени. Стенки камеры обеспечивали накопление газа в зоне взаимодействия лазерного излучения с мишенью. Игла соединялась с гелиевым баллоном с помощью силиконовой трубки. Расход гелия в этих условиях не превышал 0.251/min. Регистрация интегрального рентгеновского излучения осуществлялась ФЭУ 9107FLB со сцинтиллятором NaI и бериллиевым фильтром. Фотоэлектронный умножитель был установлен на расстоянии $30 \mathrm{~cm}$ от мишени. Рентгеновские спектры измерялись спектрометром Amptek. Положение спектрометра варьировалось в зависимости от потока рентгеновского излучения, так чтобы измерения проводились в однофотонном режиме. В случае гелиевого поддува перед спектрометром также устанавливался алюминиевый фильтр толщиной $120 \mu \mathrm{m}$. Сигнал второй гармоники, возникающей при взаимодействии сфокусированного лазерного излучения с мишенью, выделялся с помощью полосового фильтра С3С 25 и измерялся с помощью спектрометра Ocean Optics HR-400. Для изучения рентгеновской дифракции была собрана схема, состоящая из свинцовой диафрагмы с размерами $2 \times 0.3 \mathrm{~cm}$ и толщиной $12 \mathrm{~mm}$. Проходящий 


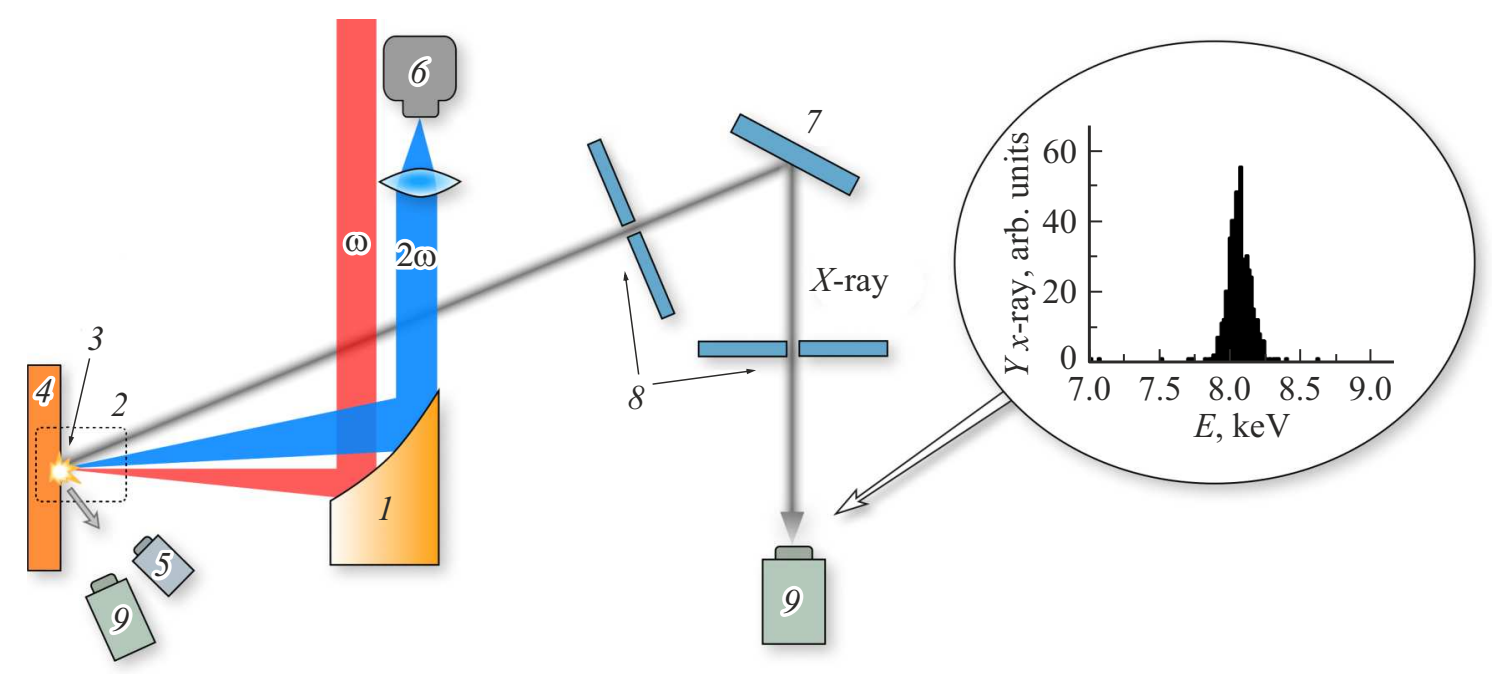

Рис. 2. Схема экспериментальной установки: 1 - внеосевая парабола, $2-$ силиконовая камера, 3 - игла, через которую осуществляется поддув гелия, 4 - медная мишень, 5 - ФЭУ, 6 - волоконный спектрометр Ocean Optics, 7 - кристалл кремния $(1,1,1), 8$ - свинцовая щель, 9 - спектрометр Amptek. На вставке - характеристический спектр, полученный после дифракции.

через щель пучок рентгеновских фотонов попадал на кристалл кремния (111), повернутый под углом Брегга к падающему излучению. Спектр отраженного излучения фиксировался спектрометром Amptek. Схема экспериментальной установки представлена на рис. 2.

\section{Экспериментальные результаты}

В проведенных экспериментах в воздухе и при гелиевом поддуве была исследована зависимость выхода характеристического рентгеновского излучения $(8 \mathrm{keV})$ от длительности импульса при фиксированной энергии $6 \mathrm{~mJ}$ (вакуумная интенсивность $4 \cdot 10^{17} \mathrm{~W} / \mathrm{cm}^{2}$ при спектрально ограниченном импульсе и $1.3 \cdot 10^{16} \mathrm{~W} / \mathrm{cm}^{2}$ при длительности 1 ps) (рис. 3,a). Получено, что выход в обоих случаях минимален при спектрально ограниченном импульсе. При увеличении длительности импульса выход нелинейно возрастает. Наблюдается небольшая асимметрия при использовании импульсов разного знака чирпа - при положительно чирпированном импульсе выход рентгеновского излучения оказывается выше примерно в 1.5 раза. В воздухе при положительно и отрицательно чирпированном импульсе выход значительно возрастает в диапазоне 30-300 fs и затем слабо увеличивается, достигая максимума при длительности около 1 ps. Амплитуда сигнала при поддуве гелия в зону взаимодействия лазерного пучка с мишенью оказывается выше примерно в 50 раз по сравнению с воздухом.

Максимум выхода рентгеновского излучения достигается также при положительно чирпированном импульсе меньшей длительности (300 fs). При использовании отрицательно чирпированного импульса максимум выхода рентгеновского излучения оказывается примерно в 1.5 раза меньше и соответствует большей длительности (500 fs).

Нами были измерены спектры характеристического рентгеновского излучения в воздухе и гелии (рис. $3, b$ ). Получено, что в воздухе максимальный выход достигает значения $4 \cdot 10^{5} \mathrm{ph} / \mathrm{pulse} / 2 \pi$ sr. В режиме поддува гелия эта величина существенно возрастает и достигает значения $2 \cdot 10^{7} \mathrm{ph} / \mathrm{pulse} / 2 \pi \mathrm{sr}$. Эффективность конверсии составляет $10^{-5}$. Данные находятся в согласии с известными результатами, полученными при гелиевом поддуве в режиме острой фокусировки [4]. Данная эффективность конверсии является рекордной для вневакуумного источника рентгеновского излучения.

Чтобы установить причину такой зависимости рентгеновского излучения от длительности импульса (и изменения вакуумной интенсивности), были выполнены измерения энергии лазерного пучка, сфокусированного в воздухе и в гелии при отсутствии мишени для оценки потерь на ионизацию газа. Также были измерены диаметры получаемых в мишени кратеров для различных длительностей лазерного импульса в воздухе и гелии. Установлено, что потери в воздухе при спектрально ограниченном импульсе достигают 70\% и падают до уровня примерно $30 \%$ при импульсе порядка 1 ps. Причем диаметр зоны оплава кратера достигает $100 \mu \mathrm{m}$ в случае положительно чирпированного импульса длительностью 1 ps, что значительно превосходит размер пучка, оцененный в отсутствие среды (вакуумные условия) перед мишенью $(\sim 10 \mu \mathrm{m})$. Поэтому хотя увеличение длительности импульса при воздействии на мишень в воздухе и приводит к уменьшению ионизации среды, однако полностью подавить влияние ионизации на дефокусировку пучка не удается. Оценка интенсивности на мишени, исходя из размера диаметра кратера, лазерной 

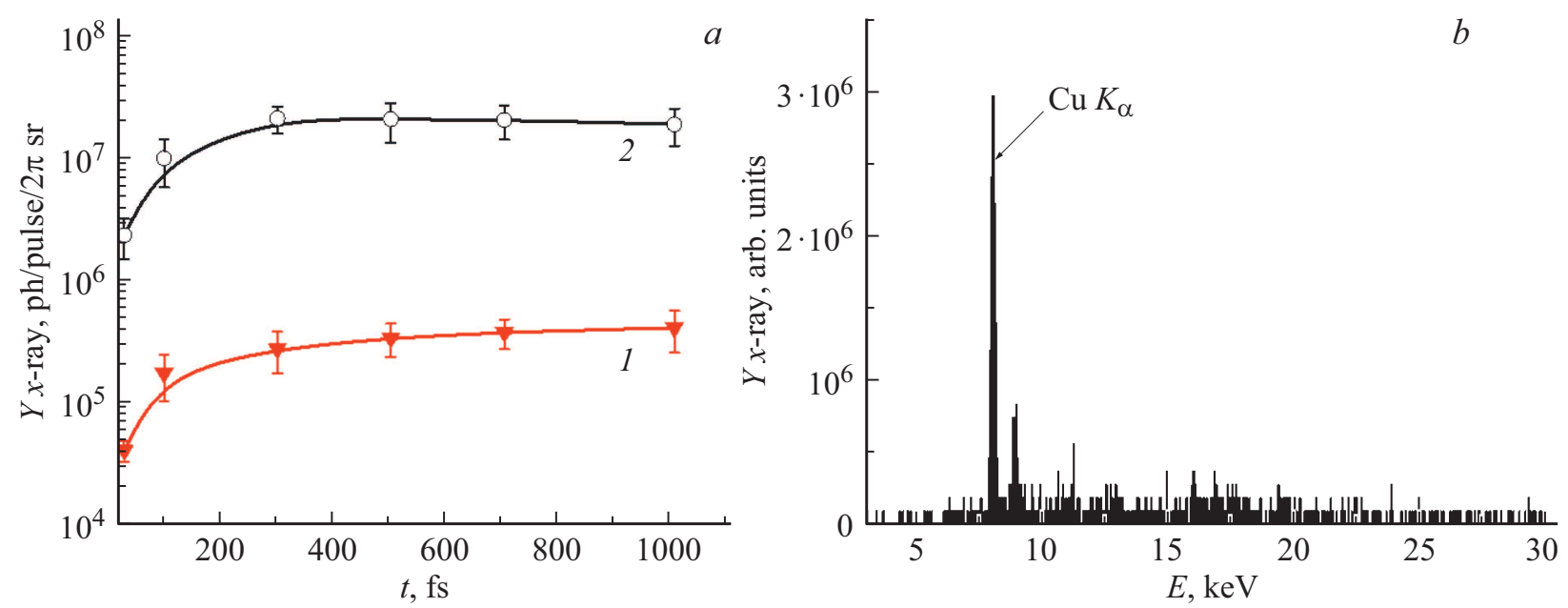

Рис. 3. Зависимость выхода рентгеновского излучения от длительности положительно чирпированного импульса в воздухе $(1)$ и гелии (2) (a). Рентгеновский спектр микроплазмы мишени при гелиевом поддуве $(b)$.
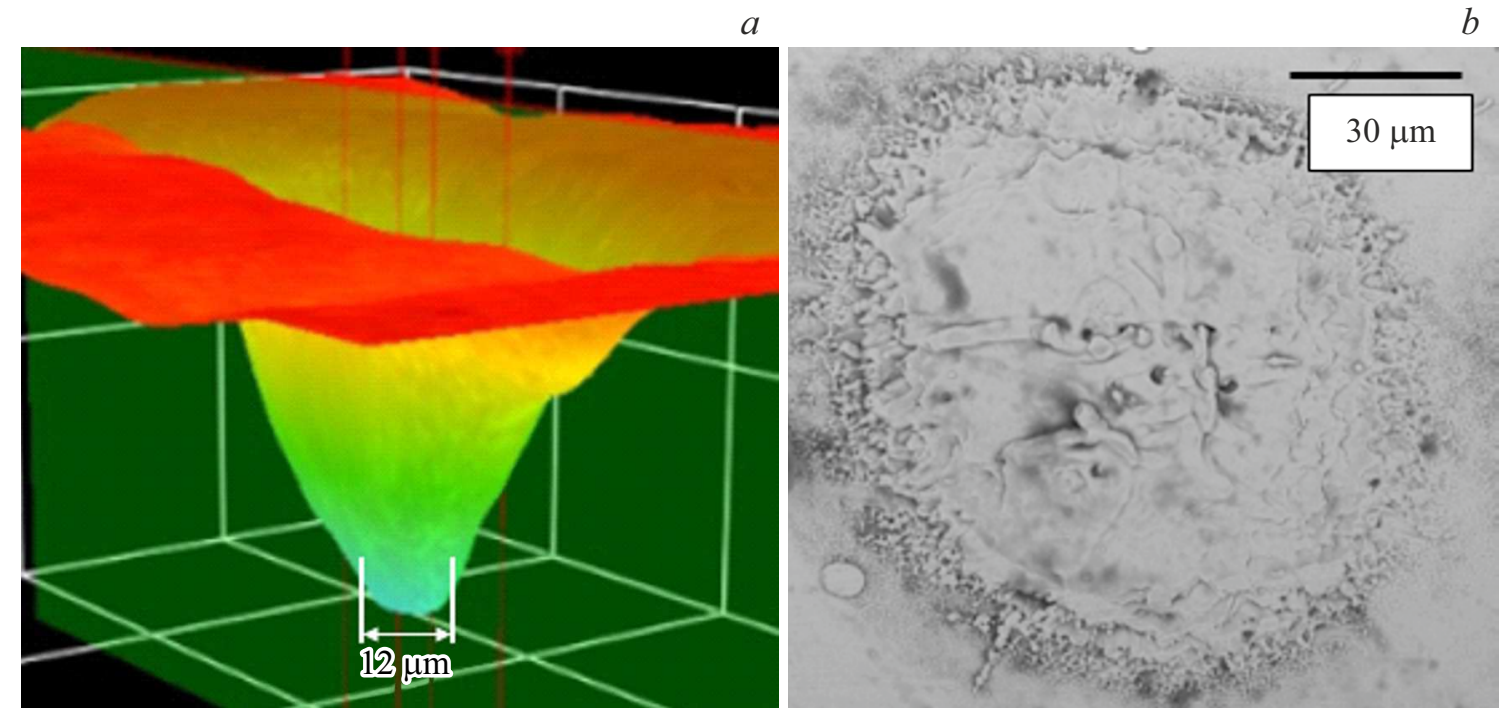

Pис. 4. (a) Изображение кратера в медной мишени, полученное при длительности лазерных импульсов $400 \mathrm{fs}$ при гелиевом поддуве с помощью 3D-микроскопа HRM-300 Series, размер ядра $12 \mu \mathrm{m}$. (b) Изображение кратера, полученное при длительности лазерных импульсов $50 \mathrm{fs}$ при гелиевом поддуве с помощью сканирующего электронного микроскопа Phenom PRO-X, диаметр оплава $100 \mu \mathrm{m}$.

энергии и длительности положительно чирпированного импульса (1 ps) оказывается ограниченной на уровне не более, чем $2 \cdot 10^{14} \mathrm{~W} / \mathrm{cm}^{2}$.

Напротив, в случае присутствия перед мишенью гелиевого слоя, имеющего больший потенциал ионизации, энергетические потери на ионизацию составляют порядка 30\% при спектрально ограниченном импульсе и спадают до уровня, при котором роль дефокусировки не существенна, при длительности порядка $300 \mathrm{fs}$. При этом диаметр кратера уменьшается со $100 \mu \mathrm{m}$ при коротком импульсе $(30-100 \mathrm{fs})$ до $12 \mu \mathrm{m}$ при длительности 300-700 fs (рис. 4). Таким образом, увеличение длительности импульса приводит к тому, что удается „обойти“ ограничение интенсивности, возникающей из- за ионизации среды, и достичь квазивакуумных условий, когда ионизация среды практически не влияет на взаимодействие лазерного импульса с мишенью.

Таким образом, при увеличении длительности импульса диаметр кратера уменьшается, а доводимая до мишени энергия растет. Поэтому увеличивается плотность энергии на мишени, которая, по-видимому, играет ключевую роль в повышении выхода рентгеновского излучения. Однако остается открытым вопрос об интенсивности на мишени, которая ответственна за появление горячих электронов, приводящих к генерации рентгеновского излучения. Отметим, что аналогичные измерения выхода рентгеновского излучения в вакуумных условиях демонстрируют спад амплитуды рентгеновского сигнала 

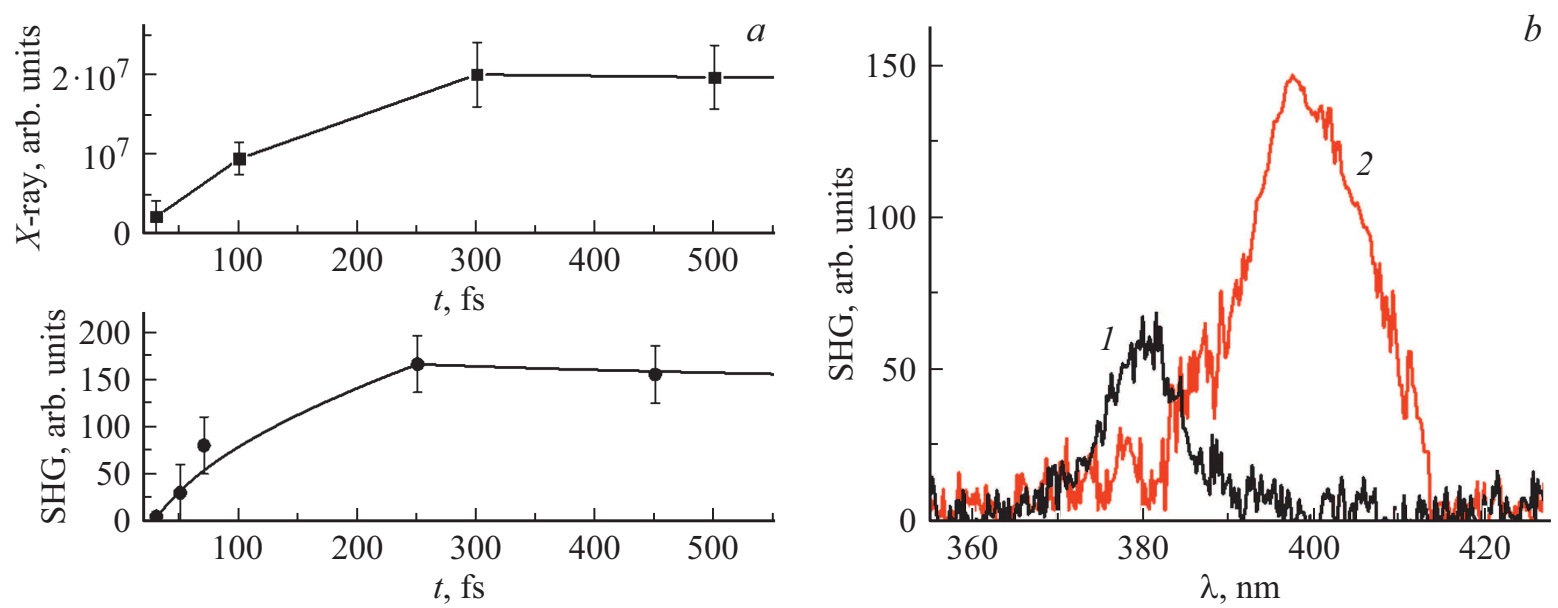

Рис. 5. (a) Зависимость сигналов рентгеновского излучения (X-ray) и второй гармоники (SHG) от длительности импульса. (b) Спектры второй гармоники при длительностях импульса $50 \mathrm{fs}(1)$ и $400 \mathrm{fs}(2)$.

с ростом длительности при сохранении энергии импульca [7].

Известно [8], что процесс воздействия интенсивного фемтосекундного лазерного излучения на мишень в воздухе может также сопровождаться генерацией второй гармоники лазерного излучения, имеющей похожую зависимость от интенсивности, что и сигнал рентгеновского излучения. Поэтому при проведении экспериментов в режиме поддува гелия одновременно измерялся сигнал второй гармоники, регистрируемый в направлении отражения от мишени. При взаимодействии спектрально ограниченного импульса с мишенью в окружении гелия наблюдалась широкополосная плазменная засветка, и сигнал второй гармоники выделить не удалось. При увеличении длительности до $50 \mathrm{fs}$ спектр второй гармоники уже можно было зарегистрировать. Ее спектр оказался сдвинутым примерно на $20 \mathrm{~nm}$ (рис. 5, b) относительно спектра, регистрируемого при длительности 400-500 fs, когда влияние плазмы уже мало. Такой сдвиг может возникать из-за изменения показателя преломления благодаря быстрой ионизации газа фронтом импульса и последующему процессу рекомбинации. По этому сдвигу можно оценить концентрацию электронов [9]:

$$
N_{e} \approx \frac{2 \pi m_{e} c^{3} \Delta \lambda}{e^{2} \lambda^{3}} \frac{\tau}{L}
$$

где $\Delta \lambda-$ сдвиг второй гармоники излучения $(\Delta \lambda \sim 20 \mathrm{~nm}), \lambda$ - длина волны излучения (в случае титан-сапфирового лазера $\lambda=0.8 \mu \mathrm{m}), \tau-$ длительность лазерного импульса $(\tau=50 \mathrm{fs}), L-$ характерная длина нелинейного взаимодействия (релеевская длина лазерного пучка, сфокусированного параболой, $L \sim 30 \mu \mathrm{m})$. Концентрация электронов $N_{e}$ оказывается равной $\sim 3 \cdot 10^{19} \mathrm{~cm}^{-3}$ и отвечает случаю полной однократной ионизации при интенсивности порядка $10^{15} \mathrm{~W} / \mathrm{cm}^{2}$ [4]. Установлено, что поведение сигнала второй гармоники в зависимости от длительности лазерного импульса подобно поведению рентгеновского излучения

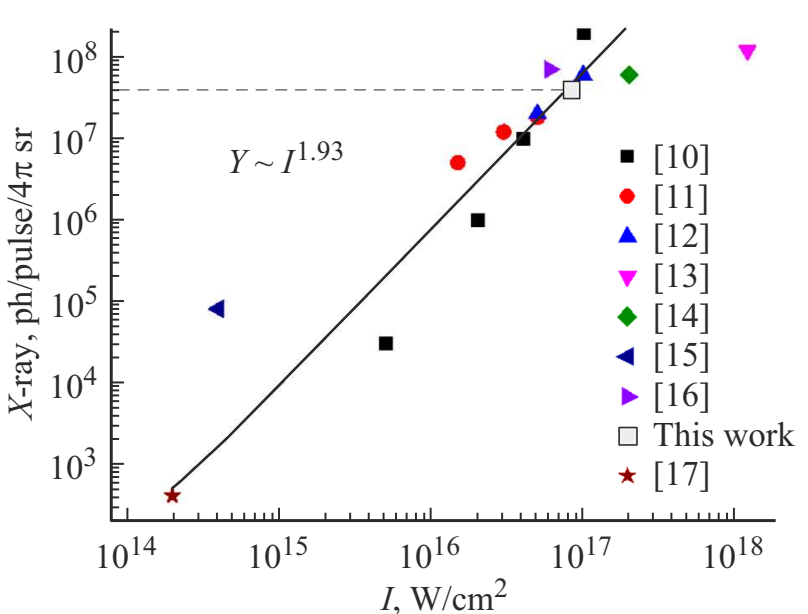

Рис. 6. Зависимость выхода характеристических рентгеновских фотонов от вакуумной лазерной интенсивности. Данные взяты из источников, указанных на рисунке. На рисунке также приведен результат настоящего эксперимента (серый квадрат).

(рис. 5,a). Так как амплитуда второй гармоники растет с увеличением длительности, то можно предположить, что также возрастает и лазерная интенсивность. Однако природа такого поведения требует дополнительного анализа.

Результаты, извлеченные из анализа диаметра кратера при длительности импульса $50 \mathrm{fs}$ (рис. 4), который зависит от потерь на ионизацию (около 30\%) и соответственно нелинейной дефокусировки, также позволяют оценить интенсивность. Она оказывается порядка $10^{15} \mathrm{~W} / \mathrm{cm}^{2}$.

На рис. 6 приведена сводная информация по выходу характеристического рентгеновского излучения в зависимости от интенсивности лазерного излучения (взята из работ [10-17] для вакуумных условий и длительности импульса 50-250 fs). На рисунке также 
приведен выход фотонов (серый квадрат), полученный в настоящем эксперименте при длительности импульса 300 fs. Зависимость выхода рентгеновского излучения от интенсивности примерно квадратичная. Примечательно, что выходу рентгеновского излучения, полученному в нашем эксперименте, соответствует диапазон интенсивности $(0.4-0.8) \cdot 10^{17} \mathrm{~W} / \mathrm{cm}^{2}[10-14]$ в зависимости от длительности импульса. Это соответствует изменению плотности энергии в пределах (4-10) $10^{3} \mathrm{~J} / \mathrm{cm}^{2}$ [10-17].

В проведенном эксперименте в гелии при длительности импульса $300 \mathrm{fs}$, диаметре дна кратера $12 \mu \mathrm{m}$ и энергии, дошедшей до дна кратера, около $5.5 \mathrm{~mJ}$ (оценена с учетом измеренных ионизационных потерь, составивших не более $10 \%$ ) можно получить значение плотности энергии около $5 \cdot 10^{3} \mathrm{~J} / \mathrm{cm}^{2}$. Это по порядку величины совпадает с оценкой плотности энергии [12], соответствующей выходу рентгеновских фотонов при длительности импульса 45 fs. При этом оцененная нами интенсивность оказывается на уровне $1.5 \cdot 10^{16} \mathrm{~W} / \mathrm{cm}^{2}$, что примерно в 5 раз ниже, чем в [12]. На наш взгляд это является свидетельством того, что возрастающая с увеличением длительности импульса плотность энергии (т.е. энерговклад) может играть значительную роль в увеличении выхода рентгеновского излучения. Для получения более ясной физической картины необходимы дополнительные исследовании.

Отметим, что согласно предположению, сделанному в [16], с увеличением длительности импульса при фиксированной лазерной энергии температура горячих электронов понижается из-за уменьшения интенсивности. Это приводит к тому, что поперечный размер области горячих электронов также сокращается. Уменьшается и размер источника характеристического излучения. Поэтому существенно, что именно размер источника определяет разрешение при рентгеноструктурном исследовании и фазово-контрастной визуализации. С другой стороны, если вложенная энергия в горячие электроны остается прежней при росте длительности импульса, то в результате количество горячих электронов увеличивается, так как вложенная энергия теперь перераспределяется в области низкоэнергетических горячих электронов [16]. Верификация этих предположений является предметом дальнейших исследований.

С использованием созданного источника характеристического рентгеновского излучения $8 \mathrm{keV}$ проведены пробные дифракционные эксперименты с кристаллическим кремнием (111). Выделена высококонтрастная линия меди в широкополосном рентгеновском спектре. Дифрагированные рентгеновские фотоны на образце, расположенном под углом Брэгга, зарегистрированы с высоким контрастом (около 100) по отношению к фону (вставка на рис. 2).

Таким образом, нами разработана схема экспериментальной установки и исследован процесс лазерноплазменной генерации рентгеновского излучения сверхкороткой длительности. Разработана методика определения интенсивности лазерного излучения на мишени и проведены соответствующие измерения. Сформулированы принципы характеристического рентгеновского излучения, генерируемого во вневакуумных условиях с использованием чирпированных лазерных импульсов, что позволяет при использовании фемтосекундного лазерного излучения в режиме высокой частоты повторений $(1 \mathrm{kHz}$ и более) получить выход, сопоставимый с потоками рентгеновских фотонов в синхротронах, но с субпикосекундной длительностью импульса.

Можно надеяться, что разработанный вневакуумный подход получения спектрально ярких потоков рентгеновских фотонов найдет применение при лабораторном решении задач, связанных с рентгеноструктурной диагностикой вещества, выполняемой с временным разрешением.

\section{Заключение}

•Предложен вневакуумный подход для эффективной лазерной генерации рентгеновского излучения, состоящий в сочетании процесса поддува гелия, обладающего высоким потенциалом ионизации и позволяющего минимизировать число индуцированных электронов, и режима чирпированных импульсов для оптимизации плотности энергии в области взаимодействия лазерного излучения с веществом.

-Установлено, что при воздействии чирпированных фемтосекундных лазерных импульсов с энергией $6 \mathrm{~mJ}$, сфокусированных $(\mathrm{NA}=0.1)$ внеосевой параболой на медную мишень в режиме гелиевого поддува в зону воздействия, выход рентгеновского излучения при длительности импульса 300-500 fs достигает максимума $2 \cdot 10^{7} \mathrm{ph} / \mathrm{pulse} / 2 \pi \mathrm{sr}$ (или $2 \cdot 10^{8} \mathrm{ph} / \mathrm{s} / 2 \pi \mathrm{sr}$ ), что является максимальным для вневакуумных условий и отвечает необходимым условиям проведения дифракционных экспериментов.

-При воздействии сфокусированных фемтосекундных лазерных импульсов на мишень, находящуюся в условиях гелиевого поддува, зарегистрирован сигнал второй гармоники в направлении зеркального отражения от мишени. При варьировании длительности лазерного импульса за счет чирпирования изменение амплитуды второй гармоники качественно соответствует динамике выхода рентгеновского излучения. По голубому сдвигу спектра гармоники при длительности $50 \mathrm{fs}$ оценена концентрация электронов в плазме, которая оказывается равной $3 \cdot 10^{19} \mathrm{~cm}^{-3}$, что отвечает условию полной однократной ионизации и соответственно интенсивности $10^{15} \mathrm{~W} / \mathrm{cm}^{2}$.

-C использованием созданного источника характеристического рентгеновского излучения $8 \mathrm{keV}$ проведены пробные дифракционные эксперименты с кристаллическим кремнием (111). Выделена высококонтрастная линия меди в широкополосном рентгеновском спектре. Дифрагированные рентгеновские фотоны на образце, 
расположенном под углом Брэгга, зарегистрированы с высоким контрастом (около 100) по отношению к фону.

\section{Благодарности}

Авторы благодарны Н.В. Минаеву за помощь в обработке изображений, Д.А. Сидорову-Бирюкову и А.В. Митрофанову за помощь по настройке лазерной системы.

\section{Финансирование}

Работа выполнена при финансовой поддержке Министерства науки и высшего образования Российской Федерации в рамках Соглашения № 075-15-2021-1358 от 12 октября 2021 г. (ID RF-0951.61321X0015).

\section{Конфликт интересов}

Авторы заявляют, что у них нет конфликта интересов.

\section{Список литературы}

[1] G. Sciaini. Appl. Sci., 9 (7), 1427 (2019). DOI: $10.3390 /$ app9071427

[2] Г.В. Фетисов. УФН, 190 (1), 2-36 (2020). DOI: $10.3367 /$ UFNr.2018.10.038435

[G.V. Fetisov. Phys.-Usp., 63 (1), 2 (2020). DOI: $10.3367 /$ UFNe.2018.10.038435]

[3] M. Afshari, P. Krumey, D. Menn, M. Nicoul, et al. Struct. Dyn., 7 (1), 014301 (2020). DOI 10.1063/1.5126316

[4] B. Hou, J. Easter, A. Mordovanakis, K. Krushelnick, J.A. Nees. Opt. Expr., 16 (22), 17695-17705 (2008). DOI 10.1364/OE.16.017695

[5] A.A. Garmatina, B.G. Bravy, F.V. Potemkin, M.M. Nazarov, V.M. Gordienko. J. Phys.: Conf. Ser., 1692 (1), 012004 (2020). DOI: 10.1088/1742-6596/1692/1/012004

[6] A.A. Garmatina, M.M. Nazarov, I.A. Zhvaniya, V.M. Gordienko, V.Y. Panchenko. Las. Phys. Lett., 16 (2), 025401 (2019). DOI 10.1088/1612-202X/aaf6a2

[7] M.M. Nazarov, P.A. Shcheglov, M.V. Chaschin, A.A. Garmatina et al. J. Phys.: Conf. Ser., 1692 (1), 012018 (2020). DOI: $10.1088 / 1742-6596 / 1692 / 1 / 012018$

[8] V.M. Gordienko, I.A. Zhvaniya, I.A. Makarov. Appl. Phys. A, 120 (2), 409-415 (2015). DOI: $10.1007 / \mathrm{s} 00339-015-9202-4$

[9] W.M. Wood, G. Focht, M.C. Downer. Opt. Lett., 13 (11), 984-986 (1988). DOI: 10.1364/OL.13.000984

[10] V. Arora, P.A. Naik, J.A. Chakera, S. Bagchi et al. AIP Adv., 4 (4), 047106 (2014). DOI: 10.1063/1.4870946

[11] M. Hada, J. Matsuo. Appl. Phys. B, 99 (1), 173-179 (2010). DOI: $10.1007 / \mathrm{s} 00340-010-3902-4$

[12] N. Zhavoronkov, Y. Gritsai, M. Bargheer, M. Woerner et al. Opt. Lett., 30 (13), 1737-1739 (2005). DOI:10.1364/OL.30.001737

[13] H. Wang, Z. Li, Z. Chen. Appl. Phys. B, 124 (9), 1-8 (2018). DOI: $10.1007 / \mathrm{s} 00340-018-7039-1$

[14] M. Iqbal, Z. Urrehman, H. Im, J.G. Son etal. Appl. Phys. B, 116 (2), 305-311 (2014).

DOI: $10.1007 / \mathrm{s} 00340-013-5691-\mathrm{z}$
[15] A. Baguckis, A. Plukis, J. Reklaitis, V. Remeikis et al. Appl. Phys. B, 123 (12), 1-7 (2017). DOI: $10.1007 / \mathrm{s} 00340-017-6868-7$

[16] R. Rathore, V. Arora, H. Singhal, T. Mandal et al. Laser and Particle Beams, 35 (3), 442-449 (2017). DOI:10.1017/S026303461700043X

[17] M. Hagedorn, J. Kutzner, G. Tsilimis, H. Zacharias. Appl. Phys. B, 77 (1), 49-57 (2003).

DOI: $10.1007 / \mathrm{s} 00340-003-1226-3$ 